\title{
Locally advanced pancreatic cancer - new therapeutic challenges
}

\author{
Michał Piątek ${ }^{1}$, Sergiusz Nawrocki ${ }^{2,3}$
}

The overall survival rate of patients with pancreatic ductal adenocarcinoma remains extremely poor, and the only potentially curative treatment is radical surgery. There are three subgroups among the patients: primary resectable, metastatic and locally advanced pancreatic cancer. The term of locally ad advanced pancreatic cancer includes borderline resectable pancreatic cancer (BRPC) and unresectable pancreatic cancer (URPC). As in the case of BRPC, the strategy of induction treatment may convert the inoperable tumour into a resectable one. As in the case of URPC, the optimal standard of treatment is unknown. Recent advances in systemic treatment such as FOLFIRINOX (5-fluorouracil, leucovorin, irinotecan, oxaliplatin) and gemcitabine plus nab-paclitaxel as well as the optimisation of local treatment such as stereotactic radiotherapy (SBRT — stereotactic body radiation therapy) should be incorporated into future trials dedicated for BRPC and URPC.

NOWOTWORY J Oncol 2016; 66, 4: 312-316

Key words: BRPC, URPC, FOLFIRINOX, gemcitabine plus nab-paclitaxel, SBRT

\section{Introduction}

Pancreatic cancer is a disease with an extremely high mortality rate - it ranks fifth among the causes of death for malignant cancers in developed countries. It is one of the few cancers in which there has been no significant progress in treatment results for the last few decades. Within the last 30 years, the chances of 5-year survival has grown from about $2 \%$ to $5 \%$ [1]. At the time of diagnosis, only $10 \%$ of patients have a resectable tumour and $60 \%$ have distant metastases while in $30 \%$ of cases the tumour is locally advanced [2].

\section{Practical clinical division into sub-groups}

Primarily the resectable group of patients is characterised by the lack of distant metastases, the lack of infiltration of the vascular structures within the celiac axis (CA), the superior mesenteric artery (SMA), the common hepatic artery $(\mathrm{CHA})$, the superior mesenteric vein (SMV), the portal vein (PV) with the admissible vein infiltration being $<180^{\circ}$ of the vessel perimeter with the possibility of reconstruction $[3,4]$. Even in the case of R0 resection, the 5 -year survival rate in this group is as low as $15-20 \%$, with median survival being about 20-24 months [5]. In the case of the R1 or R2 resection, survival is usually less than 12 months, being on a comparable level as that of the locally advanced group $[6,7]$. The rate of $\mathrm{R} 1$ resection reported in publications varied between $20 \%$ and $75 \%[8,9]$. A standard treatment after the radical resection of patients is 6-month adjuvant chemotherapy [10].

Patients with systemic involvement have the worst prognoses. The main treatment method in this group is systemic treatment, mostly with chemotherapy. The clinical benefit over treatment with 5-fluorouracil administered in the form of weekly bolus doses and the slight improvement in overall survival made gemcitabine a standard method of palliative treatment in 1997 [11]. Recent developments in the treatment of metastatic pancreatic cancer with new chemotherapeutic regimens such as gemcitabine plus nab-paclitaxel or FOLFIRINOX (5-fluorouracil, leucovorin, irinotecan, oxaliplatin) led to a significant improvement in treatment results in comparison with gemcitabine in monotherapy $[12,13]$. The comparison of FOLFIRINOX (FFX) with gemcitabine

\footnotetext{
${ }^{1}$ Clinical Oncology Division, Prof. K. Gibiński Memorial University Clinical Center, Silesian Medical University, Katowice, Poland ${ }^{2}$ Radiotherapy Division, Prof. K. Gibiński Memorial University Clinical Center, Silesian Medical University, Katowice, Poland ${ }^{3}$ Chair of Oncology and Radiotherapy, Silesian Medical University, Katowice, Poland
} 
Table I. The definition of borderline resectable pancreatic cancer according to the Intergroup trial

\begin{tabular}{llll}
\hline SMV-PV & SMA & CHA & CA \\
$\begin{array}{l}\text { Tumour - vessel interface }>180^{\circ} \\
\begin{array}{l}\text { of the vessel perimeter and/or } \\
\text { the obliteration of the vessel with } \\
\text { the possibility of performing safe } \\
\text { resection and reconstruction }\end{array}\end{array}$ & $\begin{array}{l}\text { Tumour - vessel interface }<180^{\circ} \\
\text { of the vessel perimeter }\end{array}$ & $\begin{array}{l}\text { Tumour - vessel interface } \\
\text { concerning a small segment with } \\
\text { the possibility of performing safe } \\
\text { resection and reconstruction }\end{array}$ & $\begin{array}{l}\text { Tumour }- \text { vessel interface }<180^{\circ} \\
\text { of the vessel perimeter }\end{array}$ \\
\hline
\end{tabular}

showed a sinificant increase in the objective response rate (31.6\% vs $9.4 \%)$, a significant prolongation of the survival period (11.1 vs 6.7 months) and a significant prolongation of progression-free survival (6.4 vs 3.3 months). In spite of such intensity of treatment and much larger toxicity in grade 3 and 4, no deterioration of quality of life was observed among the patients treated with FFX [13]. Irrespective of the initial doubts related to the large toxicity of FFX, this regimen was quickly introduced into clinical practice and until today it has been undergoing numerous modifications - these are mFOLFIRINOX ( $\mathrm{mFFX}$ ) which reflect the attempts to reduce toxicity accompanied with the preservation of the treatment efficacy [14-19].

The medium group of patients with a diagnosis of locally advanced neoplastic progress is characterised by the absence of distant metastases. However, some traces of the infiltration of adipose tissue around the arteries (SMA, $\mathrm{CHA}, \mathrm{CA}$ ) and/or the infiltration of veins (SMV, PV) with the involvement of $>180^{\circ}$ of the vessel perimeter and the impossibility of its reconstruction is observed $[3,4]$. This is the most heterogeneous group of patients among whom there are at the same time possibilities of radical surgery as well as palliative treatment only, and thus there are no definite standards of treatment. Given the above, it was necessary to distinguish a group of patients where the radical treatment could be possible at any stage of the therapeutic process. Eventually the locally advanced pancreatic cancer patients were divided into a group with borderline resectable pancreatic cancer (BRPC) and a group with unresectable pancreatic cancer (URPC).

\section{Borderline resectable pancreatic cancer}

The term borderline resectable pancreatic cancer has a relatively short history and it was coined because it became clear that long term survival in pancreatic cancer was only possible in the case of $\mathrm{R} 0$ resection. Other developments in this matter were defining venous resections in pancreatoduodenectomy as feasible and safe procedures, accompanied with the first reports of the possibilities of neoadjuvant treatment increasing the rate of $\mathrm{R} 0$ resections $[20,21]$.

The radiological criteria which allow one to distinguish between resectable and non-resectable cancers were described for the first time in 2001 [22]. In 2006, the National Comprehensive Cancer Network (NCCN) introduced the term "borderline resectability" to describe, in the most accurate manner, the tumours which in a limited way involve the vascular system and whose primary resection, if possible, would leave the positive surgical margins and would require the application of neoadjuvant treatment. Until today no universal definition of BRPC has been coined, which has an adverse effect on the possibilities of comparing the results of the treatment, and, as a result of which no standards in treatment have been created. The most frequently quoted definitions of BRPC were proposed by MD Anderson, America's Hepatopancreatobiliary Association (AHBPA), the Society for Surgery of the Alimentary (SSO), NCCN and by the Intergroup trial, with the latter deserving special attention, as it does not use any subjective terminology and is easy to apply to the MRI protocols (Tab. I) [4, 23-25].

A great majority of clinical studies carried out so far dealing with BRPC are retrospective, single centred and concern a small group of patients The value of these reports is limited by a diversity of the definitions of resectability and the multitude of possibilities of neoadjuvant treatment. In one such study, carried out in the MD Anderson Cancer Center, 160 (7\%) out of 2454 patients with a diagnosed pancreatic cancer were regarded as BRPC and treated with neoadjuvant therapy. The treatment was completed in 125 patients with 66 undergoing radical resection and 62 of them Ro resection (94\%). The median of overall survival in the group of 66 who completed the treatment was 40 months, whilst in the remaining 94 patients who did not go through pancreatoduodenectomy, it was 13 months ( $p<0.001$ ) [26]. Given the limitations connected with the methodology of studies concerning BRPC, the meta-analyses suggest that neoadjuvant treatment with chemotherapy or chemoradiation, based mostly on gemcitabine or 5-FU, allows for a radical surgery in $1 / 3$ of patients and the prognoses in the group of patients does not differ much from the prognoses among the primarily resectable patients $[27,28]$. In light of the new developments in the treatment of metastatic pancreatic cancer, in particular the FFX regimen, some attempts have been made to introduce it to the treatment of locally advanced pancreatic cancer, including BRPC. In 2014, the first published results on this issue concerned a prospective clinical trial with 18 patients with a diagnosis of BRPC, who first underwent neoadjuvant chemotherapy with FFX followed by a consecutive chemoradiation (50.4 $\mathrm{Gy}+$ gemcitabine or capecitabine). In the consecutive stage, 12 patients (66\%) underwent radical surgery and in all of the cases the R0 was obtained (100\%). With regards to the too short follow-up period, the final treatment results 
are missing; nevertheless 7 patients (58.3\%) out of the 12 who completed the treatment are still alive and 5 of them (41.7\%) are free from progression (18-35 months from diagnosis), whereas 6 patients who did not complete the treatment, died (6.9-17.5 months from diagnosis). It must also be stressed that the adverse event rate in the $3^{\text {rd }}$ and $4^{\text {th }}$ grade was relatively low. The most frequent complications comprised nausea/vomiting (35.7\%), neutropenia (14.3\%) and diarrhoea (14.3\%), and a large emphasis was laid on supportive care, such as, among others, the prophylactic application of granulocyte growth factor and antiemetic therapy with aprepitant - yet the toxicity of FFX did not have any negative influence on the completion of chemoradiation or mortality or peri-operative complications [29]. The largest undertaking devoted to BRPC is a multicentre pilot study, Alliance A021101, initiated by the Intergroup trial in 2013, in which the neoadjuvant treatment was based on $4 \mathrm{mFFX}$ cycles followed by a consecutive chemoradiation with capecitabine. The results of this study are hoped to be a reference point for future BRPC studies [25].

\section{Definitely non-resectable pancreatic cancer}

The distinction of BRPC from patients with a locally advanced pancreatic cancer resulted in the appearance of a group of patients with cancers with URPC - unresectable pancreatic cancer. The reports of the two carefully performed studies in this subject showed that in such cases, as opposed to BRPC, the possibility of obtaining resectability is extremely rare (1/87 and $6 / 114$ patients respectively), which, in consequence, means that long-term survival in this group is exceptionally rare [30,31]. Given the above, the main objectives of treatment in patients with URPC is improvement of quality of life and the improvement of the survival period, as the course of the disease in this group of patients differs significantly from metastatic pancreatic cancer and requires analysis in separate protocols of clinical studies [32].

The optimal therapeutic standard in URPC is highly controversial with many questions still remaining unanswered. The role of radiotherapy or chemotherapy in the treatment of URPC, now increasingly in use, as well as the effect of such treatment on overall survival is undoubtedly one of these questions. The data from the two largest trials carried out so far are contradictory. The first of them (FFCD/SFRO) compared chemoradiation (60 Gy + cisplatin and 5-fluorouracil) with gemcitabine in monotherapy. In the group of patients treated with chemoradiation, the average overall survival time was only 8.6 months, whilst in the group treated with chemotherapy alone, it was 13 months $(p<0.03)$. It must be stressed that the treatment with radiotherapy was burdened with significant toxicity, which was caused by an extensive irradiation area and a large radiation dose - and finally this was the main cause of the deterioration of treatment outcomes in this group [33]. In the second trial (ECOG 4201) chemoradiation ( $50.4 \mathrm{~Gy}+$ gemcitabine) was compared with gemcitabine in monotherapy. The average overall survival in the group of patients treated with chemoradiation was 11 months, whilst in the group treated with chemotherapy alone it was 9.2 months $(p=0.017)$. Significantly greater toxicity of treatment was observed in the chemoradiation arm: as Gl tract complications G3/4 (38 vs $14 \%, p=0.03$ ) and fatigue $G 3 / 4$ (32 vs $6 \%, p=0.006$ ) [34]. Summarising the two above trials, it seems that adding radiotherapy to chemotherapy has some insignificant effects on the improvement of overall survival, yet it is burdened with large toxicity. The final attempt to establish the role of radiotherapy in the treatment of URPC was made in the RTOG 1201 trial, currently in progress, where after an initial 3-month chemotherapy: gemcitabine + nab-paclitaxel and the exclusion of the group with the disease progression, the patients were randomly assigned to consecutive chemoradiation (50.4 Gy + capecitabine) or chemoradiation (63 Gy + capecitabine) or the continuation of chemotherapy [35].

Another issue related to the problems in the treatment of URPC is the optimal sequence of chemotherapy and chemoradiation. The recent analyses concerning the treatment with primary chemotherapy followed by consecutive chemoradiation in comparison with primary chemoradiation followed by consecutive chemotherapy point to the advantage of the first method of treatment over the latter. Primary chemotherapy carried for 2-3 months allows for the correct selection of the patients who could benefit from consecutive chemoradiation - the progression is observed in about $30 \%$ of patients after primary chemotherapy $[36,37]$

The selection of an adequate cytostatic agent for treatment with radiation, as the radio-sensitiser, is also controversial among investigators. The largest meta-analysis so far, containing 229 patients and comparing gemcitabine with 5 -fluorouracil associated with radiation, has proven the advantage of gemcitabine over 5 -fluorouracil with regards to 1 -year survival $(27.9-56.2 \%$ vs $18.3-31.6 \%, p=$ $0.03)$, however, with regards to 6-month and 2-year survival, no differences were found between the groups [38]. A recent prospective clinical study, (SCALOP trial) comparing chemoradiation based on capecitabine $\left(2 \times 830 \mathrm{mg} / \mathrm{m}^{2}\right.$ on irradiation days) as opposed to gemcitabine $(300 \mathrm{mg} /$ $\mathrm{m}^{2}$ every 7 days, 6 doses in total) preceded by a primary 3-month chemotherapy, showed a significant prolongation of the survival period in the group with capecitabine (15.2 vs 13.4 months, $p=0.01$ ); moreover, the treatment with capecitabine was characterised with a more favourable toxicity profile, both haematological (0 vs 18\%), and non-haematological one (12 vs $26 \%$ ). Contrary to gemcitabine, the administration of capecitabine during radiation could also affect the control of the disease from a systemic 
Table II. Selected clinical studies with SBRT in combination with chemotherapy (GEM, gemcitabine; 5-FU, 5-fluorouracil; GTX, gemcitabine, docetaxel, capecitabine) in locally advanced pancreatic cancer

\begin{tabular}{|c|c|c|c|c|c|c|}
\hline Study & $\begin{array}{c}\text { Number } \\
\text { of subjects }\end{array}$ & Treatment regimen & Months & $\begin{array}{c}\text { 1-year local } \\
\text { control }\end{array}$ & $\begin{array}{l}\text { Early toxicity } \\
\text { G3/4 }\end{array}$ & $\begin{array}{c}\text { Late toxicity } \\
\text { G3/4 }\end{array}$ \\
\hline Mahadevan (2010) & 36 & $8-12$ Gy x 3 + GEM & 14.3 & $78 \%$ & $8 \%$ G3 & $6 \% \mathrm{G} 3$ \\
\hline Lominska (2011) & 28 & $4-8$ Gy x 3-5 + 5-Fu/GEM & 5.9 & $86 \%$ & 0 & $7.1 \% \mathrm{G} 3$ \\
\hline Chuong (2012) & 16 & $5-10$ Gy x 5 + GTX & 15.0 & $81 \%$ & 0 & $5.3 \%$ G3 \\
\hline Tozzi (2013) & 30 & 8 Gy x 5 & 11.0 & $86 \%$ & 0 & 0 \\
\hline Gurka (2014) & 10 & 5 Gy x 5 & & & & \\
\hline$+\mathrm{GEM}$ & 12.2 & $40 \%$ & 0 & 0 & & \\
\hline Herman (multi- center, 2015) & 49 & $6.6 \mathrm{~Gy} \times 5$ & & & & \\
\hline+ GEM & 13.9 & $78 \%$ & $12.2 \%$ & $10.6 \%$ & & \\
\hline
\end{tabular}

point of view, which finally could translate into the improvement of overall survival. Irrespective of the initially small number of patients participating in the above study (74 subjects), capecitabine is now the most frequently associated with radiation in new protocols of pancreatic cancer chemotherapy [39]. The discussion of URPC treatment must point to the attempts of SBRT - stereotactic body radiation therapy alone or in combination with chemotherapy (Table II) [40-45]. The trials which have been carried out point to an effective local disease control in this method. The data concerning the safety of SBRT show very high late toxicity (ulceration, bleeding, perforation, mainly in the duodenal area), in the case of a single dose of SBRT. In the case of fractionation SBRT, the efficacy of treatment is comparable to conventional radiation with the safety of treatment kept at an acceptable level [40-48].

\section{Conclusions and future directions for development}

Resection, carried out with healthy margins, still remains the only chance for curing pancreatic adenocarcinoma. In the group of patients with locally advanced tumours, there is a distinction between the radical treatment (BRPC) and palliative approach (URPC). Preoperative treatment is not a standard approach in the treatment of pancreatic cancer, nevertheless in the case of BRPC, the application of preoperative therapy naturally seems to be the only possibility of obtaining R0 resection. Given the above, a careful qualification of the patients into specific groups is critical for the selection of the therapeutic strategy.

Distant metastases remain the main cause of the treatment failure in pancreatic cancer. The improvement of the treatment results must be found first of all in better systemic treatment. The progress in treatment obtained in metastatic pancreatic cancer, gained in such methods as FFX/mFFX or gemcitabine + nab-paclitaxel should be used for the treatment protocols of clinical studies in both BRPC and URPC $[12,13]$. Given the autopsy results pointing to the $30 \%$ rate of deaths resulting from local progression, one must not forget about the necessity of local control improvement. In spite of many controversies concerning the adequacy of irradiation in locally advanced pancreatic cancer, both conventionally fractionated chemoradiation and SBRT are frequent constituents of treatment in the protocols of clinical studies. Although there have not been any studies comparing the two methods of irradiation, SBRT is growing in frequency. This reflects the improvement of the quality of life resulting from very effective and fast pain control (less use of analgesic agents) in SBRT and a shorter treatment period than in conventional chemoradiation (1-5 days vs 5-6 weeks) [50]. There are reports which are based on molecular profiling, that point to the most probable method of disease progression. In one of the studies, it was shown that the loss of the DPC4 tumour suppressor gene was connected with an increased risk of metastatic disease, whilst its presence in the tumour tissues resulted in a more frequent local progression ( $p=0.007$ ) [49]. The possibility of predicting the method of disease progression (local vs distant) may in future be a justification for the use of local and/or systemic methods of treatment. Currently, the treatment strategy both in BRPC and URPC, apart from surgical intervention (if it is possible), comprises the combination of systemic treatment and irradiation. So far the combination of FFX or mFFX with SBRT has not been studied. Such a combination may prove to be very effective in the treatment of locally advanced pancreatic adenocarcinoma and definitely requires some further clinical research.

\section{Conflict of interest: not declared}

\section{Michał Piątek, MD}

Uniwersyteckie Centrum Kliniczne im. prof. K. Gibińskiego

Oddział Kliniczny Chemioterapii

ul. Ceglana 35, 40-952 Katowice

Recieved: 8 Mar 2016

Accepted: 29 Jun 2016 


\section{References}

1. Ries LAG, Melbert D, Krapcho $M$ et al. SEER cancer statistics review 1975-2004; based on November 2006 SEER data submision. Bethesda; MD: National Cancer Institute, 2007.

2. Jemal A, Siegel R, Ward E et al. Cancer statistics, 2009. CA Cancer J Clin 2009; 59: 225-249.

3. Edge SB, Byrd DR, Compton CC eds. AJCC cancer staging manual. $7^{\text {th }}$ ed. New York: Springer, 2010

4. Al-Hawary MM, Francis IR, Chari ST et al. Pancreatic ductal adenocarcinoma radiology reporting template: consenus statement of the Society of Abdominal Radiology and the American Pancreatic Association. Gastroenterology 2014; 146: 291-304.

5. Kuhlmann KF, de Castro SM, Wesseling JG et al. Surgical treatment of pancreatic adenocarcinoma; actual survival and prognostic factors in 343 patients. Eur J Cancer 2004; 40: 549-558.

6. Neoptolemos JP, Stocken DD, Dunn JA et al. Influence of resection margins on survival for patients with pancreatic cancer treated by adjuvant chemoradiation and/or chemotherapy in the ESPAC-1 randomized controlled trial. Ann Surg 2001; 234: 758-768.

7. Sohn TA, Yeo CJ, Cameron JL et al. Resected adenocarcinoma of the pancreas -616 patients results, outcomes and prognostic indicators. J Gastrointestinal Surg 2000; 4: 567-579.

8. Verbeke CS, Leitch $D$, Menon KV et al. Redefining the R1 resection in pancreatic cancer. Br J Surg 2006; 93: 1232-1237.

9. Raut CP, Tseng JF, Sun CC et al. Impact of resection status on pattern of failure and survival after pancreaticoduodenectomy for pancreatic adenocarcinoma. Ann Surg 2007; 246: 52-60.

10. Oettle $\mathrm{H}$, Post $\mathrm{S}$, Neuhaus $\mathrm{P}$ et al. Adjuvant chemotherapy with gemcitabine vs observation in patients undergoing curative-intent resection of pancreatic cancer: a randomized controlled trial. JAMA 2007; 297: 267-277.

11. Burris HA, Moore MJ, Andersen J et al. Improvements in survival and clinical benefit with gemcitabine as first-line therapy for patients with advanced pancreas cancer: a randomized trial. J Clin Oncol 1997; 15: 2403-2413.

12. Von Hoff DD, Ervin T, Arena FP et al. Increased survival in pancreatic cancer with nab-paklitaxel plus gemcitabine. N Engl J Med 2013; 369: 1691-1703.

13. Conroy $T$, Desseigne $F$, Ychou $M$ et al. FOLFIRINOX versus gemcitabine for metastatic pancreatic cancer. NEngl J Med 2011; 364: 1817-1825.

14. Mahaseth $\mathrm{H}$, Bruther $\mathrm{E}$, Kauch J et al. Modified FOLFIRINOX regimen with improved safety and maintained efficacy in pancreatic adenocarcinoma. Pancreas 2013; 42: 1311-1315.

15. Blazer MA, CS-Y Wu, Goldberg RM et al. Tolerability and efficacy of modified FOLFIRINOX (mFOLFIRINOX) in patients with borderline-resectable pancreatic cancer (BRPC) and locally advanced unresectable pancreatic cancer (LAURPC). J Clin Oncol 2014; 32 (Suppl 3; abstr. 275).

16. Gunturu KS, Thumar JR, Hochster HS et al. Single-institution experience with FOLFIRINOX in advanced pancreatic cancer (PC). J Clin Oncol 2012; 30 (Suppl.4; abstr. 330).

17. Metges JP, Ramee JF, Douillard JY et al.. Efficacy and safety of FOLFIRINOX in patients with metastatic pancreatic cancer. J Clin Oncol 2014; 32 (Suppl. 3; abstr. 305)

18. Alessandretti MB, Moreira RB, Brandao EP et al. 2012. Safety and efficacy of modified dose-attenuated FOLFIRINOX chemotherapy in patients over 65 years with advanced pancreatic adenocarcinoma. J Clin Oncol 2015; 33 (Suppl. 3; abstr. 468).

19. James $E$, Yao X, Cong $S$ et al. 2014. Interim analysis of a phase II study of dose-modified FOLFIRINOX (mFOLFIRINOX) in locally advanced (LAPC) and metastatic pancreatic cancer (MPC). ASCO Meeting Abstr. 32 (Suppl.3):256

20. Allema JH, Reiders ME, van Gulik TM et al. Portal vein resection in patients undergoing pancreatoduodenectomy for carcinoma of the pancreatic head. Br J Surg 1994; 81: 1642-6.

21. Spitz FR, Abbruzzese JL, Lee JE et al. Preoperative and postoperative chemoradiation strategies in patients treated with pancreaticoduodenectomy for adenocarcinoma of the pancreas. J Clin Oncol 1997; 15: 928-37.

22. Mehta VK, Fisher G, Ford JA et al. Preoperative chemoradiation for marginally resectable adenocarcinoma of the pancreas. $J$ Gastrointest Surg 2001; 5: 27-35.

23. Callery MP, Chang KJ, Fishman EK et al. Pretreatment assesment of resectable and borderline resectable pancreas cancer: expert consensus statement. Ann Surg Oncol 2009; 16: 1727-33.

24. Loyer EM, David CL, Dubrow RA et al. Vascular involvment in pancreatic adenocarcinoma: reassessment by thin-section CT. Abdom Imaging. 1996; 21: 202-6.

25. Katz MH, Marsh R, Herman JM et al. Borderline resectable pancreatic cancer: Need for standarization and methods for optimal clinical trial design. Ann Surg Oncol 2013; 20: 2787-2795.

26. Katz MH, Pisters PW, Evans DB et al. Borderline resectable pancreatic cancer: the importance of this emerging stage of disease. J Am Coll Surg 2008; 206: 833-46; discussion 846-8.
27. Assifi MM, Lu X, Eibl G et al. Neoadjuvant therapy in pancreatic adenocarcinoma: a meta-analysis of phase II trials. Surgery 2011; 150:466-473.

28. Gillen S, Schuster T, Meyer zum Büschenfelde $\mathrm{C}$ et al. Preoperative/ neoadjuvant therapy in pancreatic cancer: a systematic review and meta-analysis of response and resection percentages. PloS Med 2010; 7: e1000267.

29. Christians KK, Tsai S, Mahmoud A et al. Neoadjuvant FOLFIRINOX for borderline resectable pancreas cancer: a new treatment paradigm? Oncologist 2014; 19: 266-274

30. Kim HJ, Czischke K, Brennan MF et al. Does neoadjuvant chemoradiation downstage locally advanced pancreatic cancer? J Gastrointest Surg 2002; 6: 763-9.

31. Crane $\mathrm{CH}$, Abbruzzese $\mathrm{JL}$, Evans $\mathrm{DB}$ et al. Is the therapeutic index better with gemcitabine-based chemoradiation than with 5 -fluorouracil-based chemoradiation in locally advanced pancreatic cancer? Int J Radiat Oncol Biol Phys 2002; 52: 1293-302.

32. Phillip PA, Mooney M, Jaffe D et al. Consensus report of the National Cancer Institute clinical trials planning meeting on pancreas cancer treatment. J Clin Oncol 2009; 27: 5660-9.

33. Chauffert B, Mornex F Bonnetain F et al Phase III trial comparing initia chemoradiotherapy (intermittent cisplatin and infusional 5 -fu) followed by gemcitabine vs gemcitabine alone in patients with locally advanced non metastatic pancreatic cancer: A FFCD-SFRO study [Abstract]. JClin Oncol 2006; 24 (Suppl 18): A-4008,180s.

34. Loehrer PJ, Sr, Feng $\mathrm{Y}$, Cardenes $\mathrm{H}$ et al. Gemcitabine alone versus gemcitabine plus radiotherapy in patients with locally advanced pancreatic cancer: an Eastern Cooperative Oncology Group trial. J Clin Oncol 2011; 29: 4105-4112.

35. Ben-Josef E. A Phase II randomized trial evaluating the addition of high or standard intensity radiation to gemcitabine and nab-paclitaxel for locally advanced pancreatic cancer. RTOG 1201; version date 11/3/14.

36. Huguet $F$, Andre T, Hammel $P$ et al. Impact of chemoradiotherapy after disease control with chemotherapy in locally advanced pancreatic adenocarcinoma in GERCOR phase II and III studies. J Clin Oncol 2007; 25: 326-331. RTOG 1201; version date 11/3/14.

37. Krishnan S, Rana V, Janjan NA et al. Induction chemotherapy selects patients with locally advanced, unresectable pancreatic cancer for optimal benefit from consolidative chemoradiation therapy. Cancer 2007;110: 47-55

38. Zhu CP, Shi J, Chen YX et al. Gemcitabine in the chemoradiotherapy for locally advanced pancreatic cancer: a meta-analysis. Radiother Oncol 2011; 99: 108-113.

39. Murkherjee S, Hurt CN, Bridgewater J et al. Gemcitabine based or capecitabine-based chemoradiotherapy for locally advanced pancreatic cancer (SCALOP trial): a multicentre, randomised, phase 2 trial. Lancet Oncol 2013; 14: 317-326.

40. Mahadevan A, Jain S, Goldstein M et al. Stereotactic body radiotherapy and gemcitabine for locally advanced pancreatic cancer. Int $J$ Radiat Oncol Biol Phys 2010; 78: 735-42.

41. Lominska CE, Unger K, Nasr NM et al. Stereotactic body radiation therapy for reirradiation of localized adenocarcinoma of the pancreas. Radiat Oncol 2012; 7: 74.

42. Chuong MD, Springett GM, Freilich JM et al. Stereotactic body radiotherapy for locally advanced and borderline resectable pancreatic cancer is effective and well tolerated. Int J Radiat Oncol Biol Phys 2013; 86: 516-22.

43. Tozzi A, Comito T, Alongi $F$ et al. SBRT in unresectable pancreatic cancer: preliminary results of mono-institutional experience. Radiat Oncol 2013; 8: 148.

44. Gurka MK, Kim C, He AR et al. Stereotactic body radiation therapy (SBRT) combined with chemotherapy for unresected pancreatic adenocarcinoma. Am J Clin Oncol 2014 Aug 28 [Ahead of print].

45. Herman JM, Chang DT, Goodman KA et al. Phase 2 multi-institutional trial evaluating gemcitabine and stereotactic body radiotherapy for patients with locally advanced unresectable pancreatic adenocarcinoma. Cancer 2015; 121: 1128-37.

46. Koong AC, Le QT, Ho A et al. Phase I study of stereotactic radiosurgery in patients with locally advanced pancreatic cancer. Int J Radiat Oncol Biol Phys 2004; 58: 1017-21.

47. Koong AC, Christofferson $E$, Le OT et al. Phase II study to assess the efficacy of conventionally fractionated radiotherapy followed by a stereotactic radiosurgery boost in patients with locally advanced pancreatic cancer. Int J Radiat Oncol Biol Phys 2005; 63: 320-3.

48. Schellenberg D, Goodman KA, Lee F et al Gemcitabine chemotherapy and single-fraction stereotactic body radiotherapy for locally advanced pancreatic cancer. Int J Radiat Oncol Biol Phys 2008; 72: 678-86

49. lacobuzio-Donahue CA, Fu B, Yachida S et al. DPC4 gene status of the primary carcinoma correlates with patterns of failure in patients with pancreatic cancer. J Clin Oncol 2009; 27: 1806-1813.

50. Polistina F, Constatin G, Casamassima F et al. Unresectable locally advanced pancreatic cancer: a multimodal treatment using neoadjuvant chemoradiotherapy (gemcitabine plus stereotactic radiosurgery) and subsequent surgical exploration. Ann Surg Oncol 2010; 17: 2092-2101. 